\title{
Editorial
}

\section{'No continent is immune, including Antarctica', but National Antarctic Programmes acted collectively to avert disaster}

$\mathrm{T}$ he global reach of the COVID-19 pandemic threatened Antarctic activities, even though the continent is isolated and remote. Members of the Council of Managers of National Antarctic Programs (COMNAP) quickly recognized the extraordinary risk to Antarctic personnel in stations ill-equipped to deal with such dangerous medical conditions. Through an outstanding example of international collaboration and cooperation, COMNAP developed clear protocols for the 2020/2021 season that kept Antarctic staff safe from COVID-19.

COMNAP Members drew upon resilience and adaptability, long cultivated by working in Antarctica's extreme environment, to collectively and successfully mount an agile approach to mitigating the risks and preventing COVID-19 devastation. International cooperation through COMNAP resulted in the shared common goal of preventing the inter(intra)-continental spread of the COVID-19 disease (in)to the Antarctic Treaty area. Risk assessments and outbreak management required National Antarctic Programmes to make difficult decisions for the 2020/2021 season, which included significantly reducing the number of people deploying, emplacing quarantine and testing regimes at the gateways and implementing strict isolation protocols during travel.

COMNAP formed a COVID-19 ad hoc Subcommittee, led by Dr Tim Heitland of Germany's Alfred Wegener Institute (AWI). Lessons were learnt from the AWI-led MOSAiC project, which was in the middle of an Arctic winter project in 2019/2020 with over 600 expeditioners from 20 countries when COVID-19 started spreading. Medical advice was provided by the Joint Expert Group on Human Biology \& Medicine led by Dr Anne Hicks of the British Antarctic Survey Medical Unit, which had experience from its 2009 pandemic management workshop that focused on prevention and response to a possible influenza outbreak in Antarctica. With this expertise and through worldwide online virtual meetings with National Antarctic Programmes, common protocols for the Antarctic summer season for 2020/2021 were developed.

The COMNAP Executive Committee also held monthly online meetings to exchange information about COVID-19 and to develop prevention strategies that built on national protocols. By 16 March 2020, only 5 days after the World Health Organization (WHO) declared the pandemic, COMNAP had developed and distributed amongst its membership the 'SARS-CoV-2/COVID-19 Recommendations (non-mandatory) in the Context of Antarctic Operations'. Through the leadership of the Secretariat, COMNAP continues to review and regularly update the 'COVID-19 Outbreak Prevention \& Management Guidelines' to strengthen national efforts to prevent the introduction of the virus to Antarctica.

Regular virtual meetings allowed National Antarctic Programmes across the world to share their advanced season preparations, including their protocols for outbreak prevention, their medical resources and their response capabilities. As the global situation rapidly evolved, Antarctic nations adapted and shared lessons learnt - projects were cancelled, budgets were affected (protocol implementation costs were high) and summer science plans were impacted.

Despite the difficulties, there were successes. Education and outreach activities about Antarctica soared and all National Antarctic Programmes increased their social media and education outreach, with many reporting an unprecedented uptake of Antarctic information by public and national education outlets. Planned winter science was carried out at nearly $100 \%$ for the 2020 season and at $100 \%$ for the 2021 winter season. Countries assisted each other by retrieving or deploying scientific instrumentation, transporting cargo for upcoming seasons or assisting in the transiting of expeditioners through gateway countries. By working cooperatively, expeditioners were kept safe and Antarctic operations continued, albeit at reduced capacities and under severe restrictions.

While it was not ideal, and some may only reflect that the 2020/2021 Antarctic season resulted in reduced science, the outstanding and remarkable achievement of the international Antarctic community who worked together to overcome these extraordinary challenges should be celebrated. As the Antarctic Treaty intends, and despite such overwhelming difficulties, international cooperation succeeded in preserving the ability of National 
Antarctic Programmes to support the science in Antarctica that is so vital to understanding the future of planet Earth.

Dr Kelly K. Falkner

On detail from NSF to Science and Technology Cooperation Office, Bureau of Oceans and International Environmental and Scientific Affairs, US Department of State, Washington, DC, USA and Professor Dame Jane Francis

Director, British Antarctic Survey, Cambridge, UK 